\title{
ESTABILIDAD DIMENSIONAL Y HUMEDAD DE EQUILIBRIO EN TABLEROS DE FIBRAS (MDF) Y DE PARTÍCULAS
}

\author{
PETER NIEMZ. (*) y HERNÁN POBLETE W. (**)
}

(*) Ingeniero Civil en Maderas. Doctor Ing., Profesor Huésped. Fac. Ciencias Forestales Universidad Austral de Chile. Casilla 567. Valdivia - Chile.

(**) Ingeniero Forestal. Doctor Cs. Forestales. Profesor Titular Fac. Ciencias Forestales Universidad Austral de Chile. Casilla 567. Valdivia - Chile

\section{RESUMEN}

Se determinó la humedad de equilibrio luego del climatizado a diferentes humedades relativas del aire entre $40 \%$ y $95 \%$ en tableros de particulas y de fibras de densidad media (MDF) fabricados industrialmente. Luego del climatizado se determinaron las variaciones dimensionales longitudinales $y$ en el espesor.

Las humedades de equilibrio y las variaciones dimensionales fueron menores en los tableros $M D F$. Al reacondicionar los tableros se pudo determinar que los del tipo MDF poseen una mavor estabilidad dimensional.

Palabras Clave: Tableros MDF, Tableros de particulas, Humedad de equilibrio, Estabilidad dimensional.

\section{ABSTRACT}

Equilibrium moisture content at relative air humidities between $40 \%$ and $95 \%$ of industrial manufactured MDF and Particleboards were determinated. Longitudinat and thickness swellings were also measured.

The recorded equilibrium moisture and swelling of MDF hoards were lower than those of Particleboard. A re-conditioning of the boards showed that MDF have a higher dimensional stability.

Keywords: MDF, Particleboards, Equilibrium moisture, Dimensional Stability. 


\section{INTRODUCCIÓN}

Existen pocos estudios que traten el tema de la estabilidad dimensional y la humedad de equilibrio en tableros de fibras y de partículas.

NOACK Y SCHWAB (1986) informan que el hinchamiento longitudinal en tableros de partículas encolados con Ureaformaldehido, sufre variaciones de $0,035 \%$ por cada $1 \%$ de variación del contenido de humedad. En el caso de tableros encolados con Fenolformaldehido esta variación se reduce a $0,025 \%$ por cada $1 \%$ del contenido de humedad. Los mismos autores al determinar el hinchamiento en el espesor informan de variaciones de $0,45 \%$ y $0,70 \%$ por cada $1 \%$ de contenido de humedad, para tableros de partículas con Ureaformaldehido y Fenolformaldehido, respectivamente.

Al estudiar tableros de partículas orientadas (OSB), LANG y LOFERSKY (1995) determinaron variaciones longitudinales de $0.22 \%$ y $0,23 \%$, paralela y perpendicular a la dirección de las fibras en las caras, por cada $1 \%$ de cambio en el contenido de humedad.

EURO-MDF-BOARD (1990) establece como norma, para tableros de fibras de densidad media con espesores de 12 a $19 \mathrm{~mm}$, un aumento longitudinal máximo de 0.4 $\%$ al cambiar las condiciones climáticas de $35 \%$ de humedad relativa del aire a $85 \%$. Para el hinchamiento en el espesor EURO-MDF-BOARD (1990) establece como límite $6 \%$.

SUCHSLAND y XU (1989) determinaron que con humedades relativas superiores a $80 \%$, los tableros MDF presentan una estabilidad dimensional mayor a la de los tableros de partículas. Los mismos autores informan que la humedad de equilibrio máxima es algo inferior en MDF que en tableros de partículas (XU y SUCHSLAND, 1991).

En el presente trabajo se estudian cinco tipos de tableros MDF industriales confeccionados con Pinus radiata D.Don. encolados con Ureaformaldehido. Los tipos de tableros se diferencian por su densidad y espesor. Se incluyeron además dos tipos de tableros de partículas.

\section{MATERIAL}

Para la realización de los ensayos se consideraron tableros de fibra de densidad media (MDF) industriales del tipo liviano (MDF-L) y super liviano (MDF-SL), con densidades entre 460 y $600 \mathrm{~kg} / \mathrm{m}^{3}$. Todos los tableros fueron formados con Ureaformaldehido. A estos paneles se agregaron dos tipos de tableros de partículas.

Las claves de identificación de los tableros fueron las siguientes: 


$\begin{array}{ll}\text { MDF-SL } & \text { Tablero MDF Super Liviano (A y B) } \\ \text { MDF-L } & \text { Tablero MDF Liviano } \\ \text { TP } & \text { Tablero de Partículas }\end{array}$

Para definir las caracteristicas de los tableros se determinaron sus propiedades físicas y mecánicas. Esta caracterización se entrega en el Cuadro $\mathrm{N}^{\mathrm{o}} 1$

Cuadro $\mathrm{N}^{\circ} 1$

CARACTERIZACIÓN TECNOLÓGICA DE LOS TARLEROS.

\begin{tabular}{|c|c|c|c|c|c|}
\hline \multirow[t]{3}{*}{ TABLERO } & \multirow[t]{3}{*}{ ESPESOR } & \multirow{3}{*}{ DENSIDAD } & \multicolumn{3}{|c|}{ RESISTENCIAS } \\
\hline & & & \multicolumn{2}{|c|}{ FLEXTÓN } & \multirow[t]{2}{*}{ TRACCIÓN } \\
\hline & & & MOR & $\mathrm{MOE}$ & \\
\hline (Clave) & $(\mathrm{mm})$ & $\left(\mathrm{kg} / \mathrm{m}^{3}\right)$ & $\left(\mathrm{N} / \mathrm{mm}^{2}\right)$ & $\left(\mathrm{N} / \mathrm{mm}^{2}\right)$ & $(\mathrm{N} / \mathrm{mm} 2)$ \\
\hline $\mathrm{MDF} \cdot \mathrm{SI}$ & 15 & 549 & 31,4 & 2.316 & 0.47 \\
\hline $\mathrm{MDF}-\mathrm{SL}-\mathrm{A}$ & 18 & 495 & 32.1 & 2.335 & 0.39 \\
\hline $\mathrm{MDF} \cdot \mathrm{SL} \cdot \mathrm{B}$ & 18 & 556 & 32,2 & 2.409 & 0.79 \\
\hline MDF - 1 & 18 & 592 & 37.0 & 2.909 & 0.56 \\
\hline $\mathrm{MDF} \cdot \mathrm{SL}$ & 25 & 549 & 38.3 & 2.524 & 0.39 \\
\hline TP 16 & 16 & 598 & 20.6 & 2.289 & 0.32 \\
\hline TP 19 & 19 & 627 & 19,3 & 2.755 & 0.50 \\
\hline
\end{tabular}

TP: Tablero de particulas

MDF: Tablero de fibras de densidad media

\section{MÉTODO}

\section{Probetas}

Las determinaciones de las variaciones dimensionales longitudinales (Hinchamiento Longitudinal) se llevaron a cabo de acuerdo con la norma EN 318 en probetas de 200) $\mathrm{mm}^{*} 20 \mathrm{~mm}$. Para cada tipo de tablero se ensayaron 40 probetas de las cuales 20 se cortaron con el eje longitudinal paralelo a la dirección de producción y 20 perpendiculares al sentido de la producción.

El ensayo de Hinchamiento en el Espesor se efectuó en probetas de $50 \mathrm{~mm} * 100$ $\mathrm{mm}$. El número de probetas por tipo de tablero fue 20.

\section{Tratamiento}

Las probetas fueron tratadas en una cámara de clima marca HERAEUS a diferentes condiciones de humedad relativa del aire hasta alcanzar la humedad de equilibrio, peso constante 
Las condiciones climáticas se regularon considerando una temperatura constante de $23{ }^{\circ} \mathrm{C}$ y se varió la humedad relativa aumentándola en forma escalonada desde $40 \%$ hasta $95 \%$.

Considerando los límites señalados, en las determinaciones de humedad de equilibrio las probetas se acondicionaron pasando por los niveles $55 \%, 70 \%$ y $80 \%$. En el caso de las determinaciones de variación de las dimensiones, por dificultades técnicas del equipo de climatizado, el nivel $80 \%$ fué reemplazado por $85 \%$.

Luego de alcanzar el nivel de humedad máximo, $95 \%$, todas las probetas se climatizaron reduciendo la humedad relativa a $70 \%$ y finalmente secándolas en estufa a $103{ }^{\circ} \mathrm{C}$, hasta peso seco.

\section{Determinaciones}

La variación dimensional se calculó como el promedio de ambos sentidos de fabricación.

En el caso del Hinchamiento Longitudinal se midieron las variaciones con un equipo diseñado de acuerdo con la norma EN 318 dotado con un registrador incremental digital.

Las variaciones del Hinchamiento en el Espesor se determinaron con un registrador digital.

Las variaciones dimensionales se calcularon de acuerdo con la siguiente fórmula:

$$
\varepsilon=\frac{\left(d_{1}-d_{2}\right)}{d_{2}} * 100
$$

Donde:

$\varepsilon \quad=$ Hinchamiento $(\%)$

$d_{1}=$ Dimensión a $40,55,70,85,95,70$ y $0 \%$ hum. rel.

$\mathrm{d}_{2} \quad=$ Dimensión inicial, base a $40 \%$ hum. rel.

Con los valores de hinchamiento a $40 \%$ y $95 \%$ hum. rel. y las humedades de equilibrio respectivas, se calculó el hinchamiento porcentual por cada $1 \%$ de cambio de humedad en el tablero. Este cálculo se realizó de acuerdo con la fórmula siguiente: 


$$
\varepsilon_{\text {rel }}=\frac{\varepsilon_{95}}{\left(H_{95}-H_{40}\right)}
$$

Donde:

$\varepsilon_{\text {Tel }}=$ Hinchamiento relativo (\% por cada $1 \%$ cambio humedad del material)

$\varepsilon_{95}=$ Hinchamiento a 95 hum. rel.

$\mathrm{H}_{95}=$ Humedad de equilibrio con $95 \%$ hum. rel.

$\mathrm{H}_{40}=$ Humedad de equilibrio con $40 \%$ hum. rel.

\section{RESULTADOS Y DISCUSIÓN}

\section{Humedad de Equilibrio}

Los resultados de las determinaciones de humedad de equilibrio se presentan en el Cuadro $\mathrm{N}^{\circ} 2$.

\section{Cuadro $N^{\circ} 2$}

HUMEDAD DE EQUILIBRIO DE LOS TABLEROS Y SU DEPENDENCIA DE LA HUMEDAD RELATIVA DEL AIRE

\begin{tabular}{|l|c|c|c|c|c|}
\hline \multirow{2}{*}{$\begin{array}{c}\text { MATERIAL } \\
\text { (Clave) }\end{array}$} & \multicolumn{5}{|c|}{ HUMEDAD RELATIVA (\%) A 20 ${ }^{\circ} \mathrm{C}$} \\
\cline { 2 - 6 } & 40 & 55 & 70 & 80 & 95 \\
\hline MDF 15 SL & 6,3 & 6,8 & 7,8 & 11,5 & 17,6 \\
MDF 18 SL-A & 6,6 & 7,1 & 8.2 & 11,4 & 17.4 \\
MDF 18 SL-B & 6,3 & 7,0 & 8,1 & 11,5 & 17,1 \\
MDF 18 L & 7,1 & 7,6 & 8,4 & 11,3 & 16,3 \\
MDF 25 SL & 6,9 & 7,6 & 7,8 & 10,9 & 16,6 \\
TP 16 & 7,3 & 8.0 & 10.0 & 13,7 & 19,0 \\
TP 19 & 7,3 & 7,9 & 8.9 & 13,3 & 19,4 \\
\hline
\end{tabular}

TP : Tablero de particulas

MDF : Tablero de fibras de densidad media

En general los resultados demuestran que la humedad de equilibrio alcanzada por los tableros es marcadamente inferior a la alcanzada en estudios paralelos con madera sólida de Pinus radiata. Esta diferencia puede deberse al tratamiento térmico que sufren las partículas y las fibras de los tableros durante el desfibrado y secado. Determinaciones en este sentido han sido efectuadas anteriormente, demostrándose que el tiempo y la temperatura de secado afectan la capacidad de las partículas para retener agua (POBLETE, 1984).

El análisis del comportamiento de los paneles permite observar que al climatizar en una humedad relativa de $\mathbf{8 0} \%$ los tableros de partículas alcanzan una humedad de equilibrio aproximadamente $2 \%$ mayor a la de los tableros de fibras. Esta diferencia se 
daría por la permanencia de lignina en la superficie de las fibras durante el desfibrado termomecánico en la producción de tableros de fibras. La lignina tiene una humedad de equilibrio menor al resto de los componentes principales de la madera (KOLLMANN y SCHNEIDER, 1963).

\section{Variaciones Dimensionales en el Largo}

En el Cuadro $\mathrm{N}^{\circ} 3$ se presentan los resultados del hinchamiento longitudinal, calculados en base al largo de las probetas en una humedad relativa de $40 \%$.

Cuadro $\mathrm{N}^{\circ} 3$

HINCHAMIENTO LONGITUDINAL CALCULADO EN BASE A UNA HUMEDAD RELATIVA DEL AIRE DE $40 \%$

\begin{tabular}{|l|c|c|c|c|c|c|c|}
\hline \multirow{2}{*}{$\begin{array}{c}\text { MATERIAL } \\
\text { (Clave) }\end{array}$} & \multicolumn{7}{|c|}{ HUMEDAD RELATIVA DEL AIRE (\%) } \\
\cline { 2 - 7 } & 40 & 55 & 70 & 85 & 95 & 70 & 0 \\
\hline MDF 15 SL & 0 & 0,04 & 0,15 & 0,18 & 0,19 & 0,10 & $-0,32$ \\
MDF 18 SL-A & 0 & 0,06 & 0,13 & 0.17 & 0,19 & 0,08 & $-0,35$ \\
MDF 18 SL-B & 0 & 0,03 & 0,11 & 0,14 & 0,16 & 0,05 & $-0,32$ \\
MDF 18 L & 0 & 0,05 & 0.10 & 0.18 & 0.19 & 0,08 & $-0,38$ \\
MDF 25 SL & 0 & 0,06 & 0,15 & 0,17 & 0,22 & 0,08 & $-0,35$ \\
\hline Promedio MDF & 0 & 0,05 & 0,13 & 0,17 & 0,19 & 0,08 & $-0,34$ \\
\hline TP 16 & 0 & 0,06 & 0,18 & 0,27 & 0,38 & 0,21 & $-0,28$ \\
TP 19 & 0 & 0,04 & 0,16 & 0,23 & 0,38 & 0,22 & $-0,29$ \\
\hline Promedio TP & 0 & 0,05 & 0,17 & 0,25 & 0,38 & 0,22 & $-0,29$ \\
\hline
\end{tabular}

TP : Tablero de particulas

MDF : Tablero de fibras de densidad media

En general los valores de hinchamiento son inicialmente, hasta un $55 \%$ de humedad relativa, iguales en ambos tipos de tablero. A partir de este punto los hinchamientos de MDF resultaron menores a los de tableros de partículas (ver Figura $\mathrm{N}^{0}$ 1). 


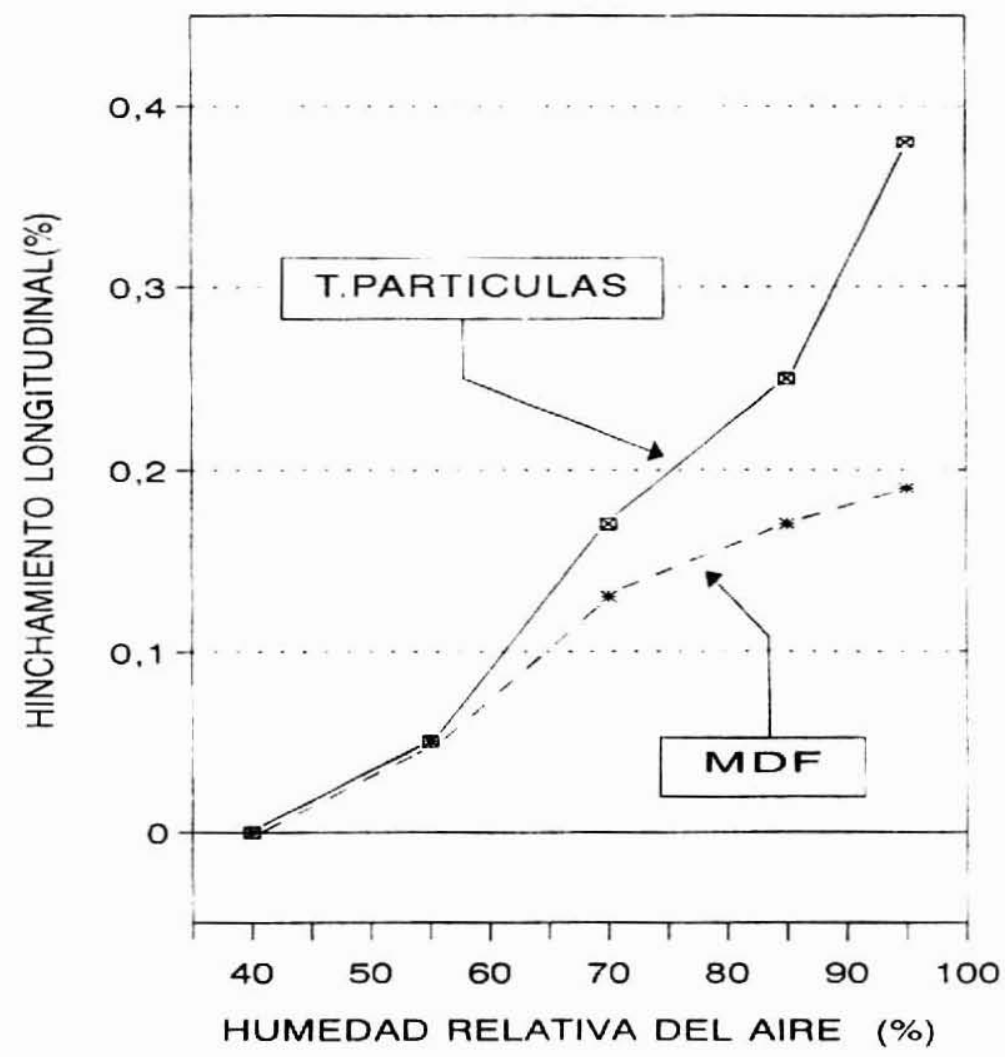

Figura $N^{\circ}$ 1. EFECTO DE LA HUMEDAD RELATIVA SOBRE EL HINCHAMIENTO LONGITUDINAL (PROMEDIO DE TODOS LOS TABLEROS)

El hinchamiento longitudinal de los tableros MDF con una humedad relativa del aire a $85 \%$ es de $0,17 \%$, lo que permite aseverar que los tableros cumplen con la exigencia de la norma EMB $(0,4 \%)$. Para el caso de los tableros de partículas no existe una exigencia de estas características.

Al reducir la humedad de equilibrio de $95 \%$ a $0 \%$, se pudo determinar que los tableros MDF presentan un hinchamiento longitudinal con mayor capacidad de recuperación que los de partículas (ver Cuadro $\mathrm{N}^{\mathrm{o}} 3$ ).

Las diferencias entre tableros MDF y de particulas son atribuibles a diferencias estructurales y a la estabilización del hinchamiento de la madera lograda a través del tratamiento térmico con vapor en el desfibrado. Lo anterior queda demostrado por los trabajos de BURMESTER (citado por LEXIKON DER HOLZTECHNIK, 1988) quien desarrolló un proceso para una estabilización dimensional de tableros de partículas a 
través de un tratamiento térmico de las partículas húmedas. También es conocido que especies de secado dificil, por ejemplo Coigie (Nothofagus dombeyi), a través de un pretratamiento con agua caliente o vapor se estabilizan, disminuyendo las posibilidades de colapso y aparición de grietas (DIAZ-VAZ y POBLETE, 1989). En este sentido es importante el trabajo de MATEJAK (1982) quien demostró que con tratamientos cíclicos de humectación se logran disminuir las variaciones dimensionales en madera de Fagus sylvatica.

La relación entre humedad del tablero e hinchamiento longitudinal puede ser representada con exactitud por una regresión lineal de primer grado (ver Cuadro $\mathrm{N}^{\circ} 4$ ), cuya ecuación es la siguiente:

$$
H_{1}=A+B^{*} H r
$$

Donde:

$$
\begin{array}{ll}
\mathrm{H}_{1} & =\text { Hinchamiento longitudinal (\%) } \\
\text { A y B } & =\text { constantes } \\
\mathrm{H}_{\mathrm{r}} & =\text { Humedad rel. aire (\%) }
\end{array}
$$

Cuadro $\mathrm{N}^{0} 4$

HINCHAMIENTO LONGITUDINAL: REGRESIONES

\begin{tabular}{|l|c|c|c|}
\hline \multirow{2}{*}{ Material } & \multicolumn{2}{|c|}{ CONSTANTES } & COEFICIENTE \\
& CORRELACION \\
\cline { 2 - 3 } & A & B & \\
\hline MDF 15 SL & $-0,148$ & 0,0038 & 0.96 \\
MDF 18 SLA & $-0,133$ & 0.0035 & 0,99 \\
MDF 18 SL-B & -0.125 & 0.0031 & 0.98 \\
MDF 18 L & $-0,149$ & 0.0036 & 0.99 \\
MDF 25 SL & $-0,155$ & 0,0039 & 0,99 \\
Promedio MDF & -0.140 & 0.0036 & 0,99 \\
TP 16 & $-0,125$ & 0,0031 & 0,98 \\
TP 19 & $-0,298$ & 0.0068 & 0,99 \\
Promedio TP & $-0,298$ & 0,0068 & 0,99 \\
\hline
\end{tabular}

TP : Tablero de particulas

MDF : Tablero de fibras de densidad media

Hinchamiento calculado en base a una humedad relativa del aire $=40 \%$

En el ensayo de hinchamiento longitudinal se tuvo en consideración la dirección de fabricación. Los resultados demostraron que debido a que existe una orientación parcial de las particulas y fibras. el hinchamiento longitudinal es menor en el sentido paralelo a la dirección de fabricación. Las diferencias entre ambas mediciones han sido representadas como proporción (Perpendicular/Paralela) en el Cuadro $\mathrm{N}^{\circ} 5$. 
La dependencia del hinchamiento del sentido de fabricación se fundamenta en la anisotropia de la madera y su menor hinchamiento en el sentido longitudinal.

La existencia de una relación con la dirección de fabricación ha sido determinada por numerosos autores para el caso del módulo de elasticidad y el módulo de rotura en flexión, donde estas propiedades resultan alrededor de $10 \%$ mas altas cuando el eje de la probeta queda paralelo al sentido de fabricación (NIEMZ, ROLLERI, BARRADIT. 1995).

Los resultados obtenidos demuestran que las diferencias entre los hinchamientos longitudinales paralelo y perpendicular a la dirección de fabricación, tienen variaciones importantes entre los tableros MDF. En el caso de los tableros de partículas los resultados muestran un comportamiento homogéneo, donde los hinchamientos perpendiculares son treinta veces mayores a los paralelos.

\section{Cuadro $\mathrm{N}^{\circ} 5$ \\ PROPORCIÓN ENTRE HINCHAMIENTO LONGITUDINAL PERPENDICULAR Y PARALELO A LA DIRECCIÓN DE FABRICACIÓN}

\begin{tabular}{|l|c|}
\hline \multicolumn{1}{|c|}{ MATERIAL } & $\begin{array}{c}\text { PERPENDICULAR / PARALELO } \\
(\%)\end{array}$ \\
\hline MDF 15 SL & 1.6 \\
MDF 18 SL-A & 3.3 \\
MDF 18 SL-B & 11.6 \\
MDF 18 L & 2.2 \\
MDF 25 SL & 12.0 \\
TP 16 & 33.0 \\
TP 19 & 37.0 \\
\hline
\end{tabular}

TP : Tablero de particulas

MDF : Tablero de fibras de densidad media

\section{Variaciones Dimensionales en el Espesor}

Los resultados de las mediciones del hinchamiento en el espesor se presentan en el Cuadro $\mathrm{N}^{\circ} 6$. Los resultados demuestran que el hinchamiento del espesor es mayor en los tableros de partículas que en los MDF. 
Cuadro $N^{\circ} 6$

HINCHAMIENTO EN EL ESPESOR CALCULADO EN BASE AL ESPESOR CON UNA HUMEDAD RELATIVA DE $40 \%$

\begin{tabular}{|l|c|c|c|c|cc|}
\hline \multirow{2}{*}{$\begin{array}{c}\text { MATERIAL } \\
\text { (Clave) }\end{array}$} & \multicolumn{5}{|c|}{ HINCHAMIENTO EN EL ESPESOR (\%) BASE 40\% H.R.A. } \\
\cline { 2 - 7 } & 40 & 55 & 70 & 85 & 95 & 0 \\
\hline MDF 15 SL & 0 & 0,20 & 1,50 & 3,80 & 8,90 & $-2,00$ \\
MDF 18 SL-A & 0 & 0,20 & 1,30 & 3,20 & 8,20 & $-2,70$ \\
MDF 18 SL-B & 0 & 0,20 & 1,50 & 3,40 & 8,00 & $-2,10$ \\
MDF 18 L & 0 & 0,30 & 1,40 & 2,80 & 6,50 & $-2,80$ \\
MDF 25 SL & 0 & 0,30 & 1,50 & 3,30 & 6,90 & $-1,40$ \\
\hline Promedio MDF & 0 & 0,24 & 1,44 & 3,30 & 8,30 & $-2,14$ \\
\hline TP 16 & 0 & 0,20 & 1,90 & 4,30 & 9,30 & 3,90 \\
TP 19 & 0 & 0,30 & 1,70 & 3,30 & 10,80 & 2,30 \\
\hline Promedio TP & 0 & 0,25 & 1,80 & 3,80 & 10,05 & 3,09 \\
\hline
\end{tabular}

TP : Tablero de particulas

MDF : Tablero de fibras de densidad media

Los tableros MDF registraron un hinchamiento del espesor de 3,3\% al aumentar la humedad relativa a $85 \%$. De acuerdo con EURO-MDF-BOARD (1990) el hinchamiento máximo no debe superar $6 \%$ por lo que los tableros cumplen con esta exigencia. Para los tableros de partículas no existe un requisito de este tipo.

De los resultados es importante destacar que los tableros de fibra (MDF) luego del secado, a $0 \%$ de humedad, alcanzan un espesor menor que el inicial (determinado con $40 \%$ de humedad relativa). Por el contrario, en los tableros de particulas se registra un hinchamiento irreversible mayor al inicial. Esta diferencia corresponde al fenómeno denominado Springback (ver Figura $\mathrm{N}^{\circ} 2$ ).

Las diferencias entre ambos tipos de tableros se deben a que con el climatizado a altas humedades relativa. se provoca una destrucción parcial de las uniones entre las partículas. 


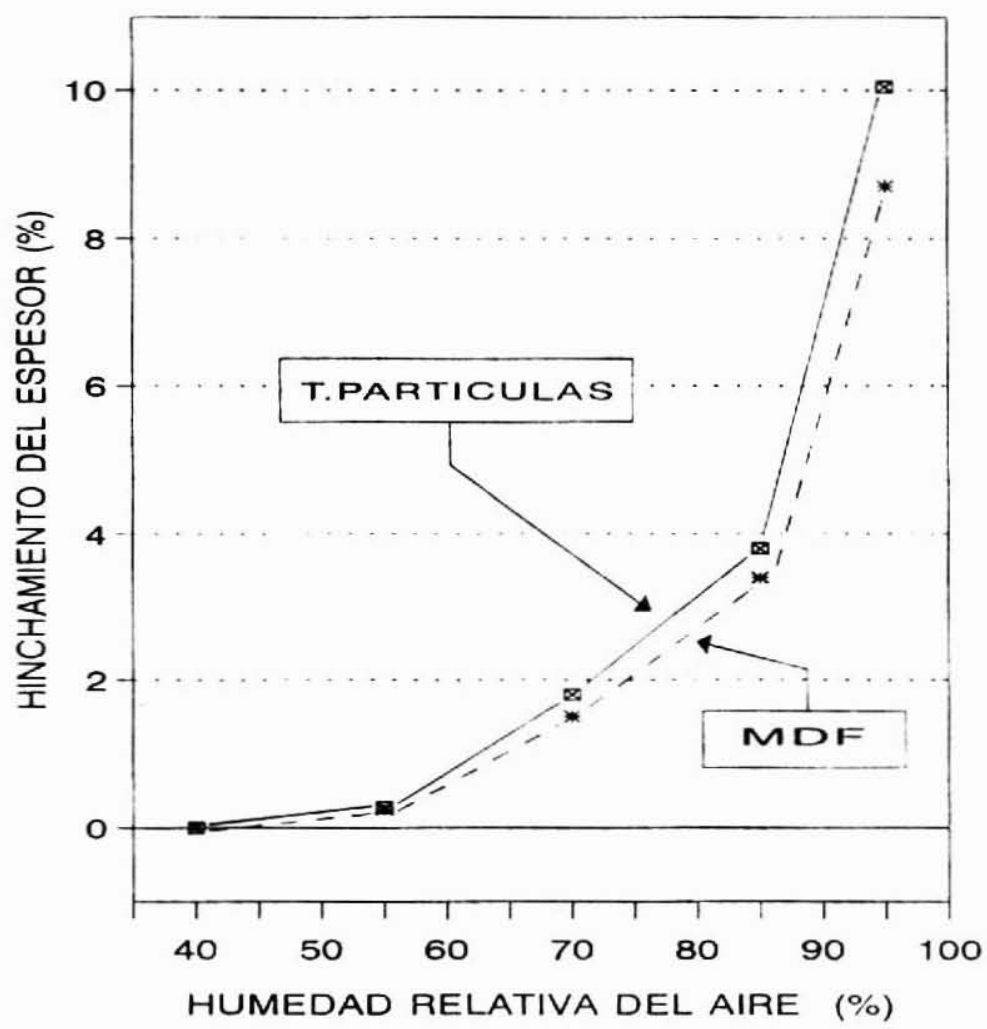

Figura $N^{\circ}$ 2: EFECTO DE LA HUMEDAD RELATIVA SOBRE EL HINCHAMIENTO DEL ESPESOR (PROMEDIO DE TODOS LOS TABLEROS)

Con los valores de hinchamiento en el espesor se efectuó un análisis de regresión. obteniéndose los resultados que se presentan en el Cuadro $N^{\circ} 7$. De los resultados de este análisis se debe destacar los altos valores de correlación registrados para todos los tipos de tableros ensayados.

Las ecuaciones calculadas son de primer grado y corresponden al siguiente modelo:

$$
H_{e}=A+B^{*} H r
$$

Donde:

$\mathrm{H}_{1} \quad=$ Hinchamiento longitudinal (\%)

A y $\mathrm{B}=$ constantes

$\mathrm{H}_{\mathrm{r}} \quad$ = Humedad rel. aire (\%) 
Cuadro $\mathrm{N}^{0} 7$

HINCHAMIENTO DEL ESPESOR: REGRESIONES

\begin{tabular}{|l|c|c|c|}
\hline \multirow{2}{*}{ MATERIAL } & \multicolumn{2}{|c|}{ CONSTANTES } & \multirow{2}{*}{$\begin{array}{c}\text { COEFICIENTE } \\
\text { CORRELACIÓN }\end{array}$} \\
\cline { 2 - 3 } & $\mathrm{A}$ & $\mathrm{B}$ & 0,99 \\
MDF 15 SL & $-0,002158$ & 1,092602 & 0,99 \\
MDF 18 SL-A & $-0,001425$ & 1,096251 & 0,97 \\
MDF 18 SL-B & $-0,002615$ & 1,089073 & 0,99 \\
MDF 18 L & $-0,005302$ & 1,078056 & 0,99 \\
MDF 25 SL & $-0,003203$ & 1,087660 & 0,99 \\
\hline Promedio MDF & $-0,002554$ & 1,089660 & 0,98 \\
\hline TP 16 & $-0,002510$ & 1,092036 & 0,98 \\
TP 19 & $-0,003370$ & 1,087814 & 0,98 \\
\hline Promedio TP & $-0,002925$ & 1,089910 & \\
\hline
\end{tabular}

TP : Tablero de particulas

MDF : Tablero de fibras de densidad media

Hinchamiento calculado en base a una humedad relativa del aire $=40 \%$

\section{Variaciones Dimensionales en Relación al Cambio de Humedad de Equilibrio}

Con los valores obtenidos en los ensayos de hinchamiento longitudinal y del espesor se calculó la variación de las dimensiones, en porcentaje, por cada $1 \%$ de variación de la humedad de equilibrio del tablero (ver Cuadro $\mathrm{N}^{\circ} 8$ ).

Este parámetro ha sido determinado en estudios anteriores señalándose que en tableros de partículas encolados con Ureaformaldehido la variación longitudinal fué $0.035 \%$ y alcanza a $0.45 \%$ en el espesor (NOACK Y SCHWAB. 1986).

En el presente estudio el hinchamiento longitudinal de los tableros de partículas fue menor a lo citado por la literatura, mientras que en el caso del hinchamiento en el espesor los valores son más altos. Las diferencias pueden deberse al uso de aditivos con el adhesivo (por ejemplo hidrófobos) o a la aplicación de adhesivos distintos a los de otros estudios, ya sea en la cantidad, tipo o calidad. 


\section{Cuadro No 8 \\ HINCHAMIENTO POR CADA $1 \%$ DEL CAMBIO DE HUMEDAD DE EQUILIBRIO DEL TABLERO.}

\begin{tabular}{|l|c|c|}
\hline \multirow{2}{*}{ MATERIAL } & \multicolumn{2}{|c|}{ HINCHAMIENTO (\% POR CADA 1\%) } \\
\cline { 2 - 3 } & LONGITUDINAL & ESPESOR \\
\hline MDF 15 SL & 0,017 & 0,79 \\
MDF 18 SL-A & 0,018 & 0,76 \\
MDF 18 SL-B & 0,015 & 0,74 \\
MDF 18 L & 0,020 & 0,70 \\
MDF 25 SL & 0,023 & 1,02 \\
\hline Promedio MDF & 0,019 & 0,80 \\
\hline TP 16 & 0,031 & 0,77 \\
TP 19 & 0,032 & 0,92 \\
\hline Promedio TP & 0,032 & 0,84 \\
\hline
\end{tabular}

TP : Tablero de particulas

MDF : Tablero de fibras de densidad media

No existen investigaciones que estudien esta relación del hinchamiento con la humedad de equilibrio en tableros de fibras de densidad media (MDF). Por lo anterior no se tienen valores que sirvan de referencia para estos tableros.

\section{BIBLIOGRAFÍA}

Diaz-Vaz, J.E.; Poblete, H. 1989. Pretratamiento de Madera para Muebles. CORFO. Fondo de Desarrollo Productivo. Corporación de Fomento de la Producción. 67 p.

EN-318. 1993. Fasserplatten. Bestimmung von Massaenderungen in Verbindung mit Aenderungen der relativen Luffeuchte.

Euro-Mdf-Board. 1990. Mitteldichte Faserplatten (MDF): Definition, Prufbedingungen und Qualitătsanforderungen. Industrienorm. $30 \mathrm{p}$.

Kollmann, F.; Shneider, A. 1963. Über das Sorptionsverhalten WWärmebehandelter Hölzer. Holz als Roh- und Werkstoff 21 (3): 77-85.

Lang, E.; Lofersky, J. 1995. In-plane Hygroscopic Expansion of Plywood and Oriented Strandboard. Forest Products Journal 45(4): 67-71

Lexikon Der Holztechnik. 1988. 3-Edición. VEB Fachbuchverlag. Leipzig. 961 p.

Matejak, M. 1982. Einfluss des Trocknens von Holz auf den Verlauf seiner Sorptionsisothermen. Holzforschung und Holzverwertung 35 (4): 77-85.

Niemz, P.; Rolleri, A.; Barradit, E. 1995. Untersuchungen zur Schallausbreitungsgeschwindigkeit in Spanplatten un MDF und deren Korrelation mit den elastomechanischen Eigenschaften. Holz- Zentralblatt. Im Druck. 
Noack, D.; Schwab, E. 1986. En: von Halaz, R.; Scheer, R.: Holzbautaschenbuch. Ernst-Verlag fur Architektur u. Technische Wissenschaften. Berlin.

Poblete, H. 1984. Verănderungen in Holzspanen wăhrend der Trocknung und des Pressens zu Holzspanplatten. Disertación para optar a Doctorado. U. de Gơttingen. $157 \mathrm{p}$.

Suchsland, O.; Xu, H. 1989. On the Measurement of Linear Expansion of Particleboard and Medium Density Fiberboard. Forest Products Journal 39 (6): 39-42.

Xu, H.; Suchsland, O. 1991. The Expansion Potential: A New Evaluator of the Expansion Behavior of Wood Composites. Forest Products Journal 41 (6): 39-42. 